\title{
Optical design and performance of the SuperCam instrument for the Perseverance rover
}

Pernelle Bernardi, Laurent Parès, Raymond Newell, Tony Nelson, Olivier Gasnault, et al.

Pernelle Bernardi, Laurent Parès, Raymond Newell, Tony Nelson, Olivier Gasnault, Jean-Michel Réess, Vishnu Schridar, Ivair Contijo, Adriana ReyesNewell, Glenn E. Peterson, Chip Legett IV, Bruno Dubois, Scott H. Robinson, "Optical design and performance of the SuperCam instrument for the Perseverance rover," Proc. SPIE 11852, International Conference on Space Optics - ICSO 2020, 118521H (11 June 2021); doi: 10.1117/12.2599243

SPIE Event: International Conference on Space Optics - ICSO 2021, 2021, Online Only 


\section{International Conference on Space Optics-ICSO 2020}

Virtual Conference

30 March-2 April 2021

Edited by Bruno Cugny, Zoran Sodnik, and Nikos Karafolas
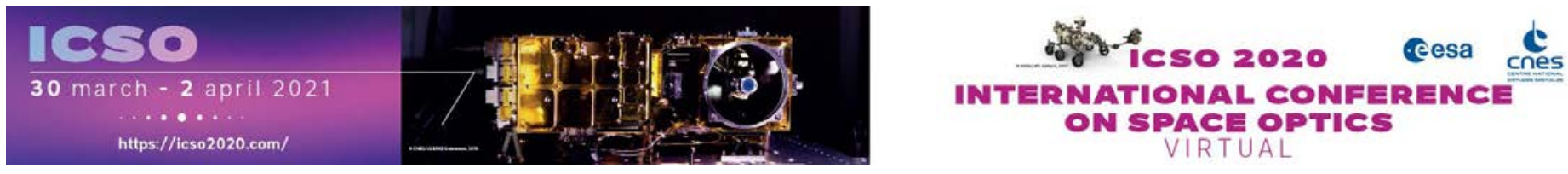

\section{Optical design and performance of the SuperCam instrument for the Perseverance rover}

\section{Cesa isoporecestings denes}




\title{
Optical design and performance of the SuperCam instrument for the Perseverance rover
}

${\text { Pernelle Bernardi*a }{ }^{*} \text { Laurent Parès }}^{\mathrm{b}}$, Raymond Newell ${ }^{\mathrm{c}}$, Tony Nelson ${ }^{\mathrm{c}}$, Olivier Gasnault ${ }^{\mathrm{b}}$, Jean-Michel Reess ${ }^{\mathrm{a}}$, Vishnu Schridar $^{\mathrm{d}}$, Ivair Contijo ${ }^{\mathrm{d}}$, Adriana Reyes-Newell ${ }^{\mathrm{c}}$, Glen Peterson ${ }^{\mathrm{c}}$, Chip Legett IV ${ }^{\mathrm{c}}$, Bruno Dubois ${ }^{\mathrm{e}}$, Scott Robinson ${ }^{\mathrm{c}}$, Philippe Caïs ${ }^{\mathrm{f}}$, Muriel Deleuze ${ }^{\mathrm{g}}$, Yves André ${ }^{\mathrm{g}}$, André Debus ${ }^{\mathrm{g}}$, Sylvestre Maurice ${ }^{\mathrm{b}}$, Roger Wiens ${ }^{\mathrm{c}}$ and the SuperCam team.

aLESIA, Observatoire de Paris, Université PSL, CNRS, Sorbonne Université, Université de Paris, 5 place Jules Janssen 92190 MEUDON, France ; 'IRAP, Université Paul Sabatier III, CNRS, 9 avenue du Colonel Roche, 31400 Toulouse, France ; 'Los Alamos National Laboratory, Los Alamos NM 87545, USA; 'NASA JPL, 4800 Oak Grove Dr, Pasadena, CA 91109, USA; ' OMP, 14 avenue Edouard Belin, 31400 Toulouse, France; ${ }^{\text {LAB }}$, Université de Bordeaux, CNRS, allée Geoffroy Saint-Hilaire, 33600 Pessac, France ; ${ }^{g}$ CNES, 18 Avenue Edouard Belin, 31400 Toulouse, France

*pernelle.bernardi@obspm.fr; phone+3314507 77 18; https:/lesia.obspm.fr

\begin{abstract}
On the $30^{\text {th }}$ of July 2020, NASA launched the Mars2020 mission. This mission, very similar to Mars Science Laboratory, consists in landing the Perseverance rover on the Martian surface in order to characterize the geology and history of Jezero Crater landing site, investigate Mars habitability, seek potential bio-signatures, cache samples for a future return to Earth, and demonstrate in-situ production of oxygen needed for human exploration.

The SuperCam instrument, an improved version of the ChemCam instrument on Curiosity rover, implements a remote micro-scale characterization of the mineralogy and elemental chemistry of the Mars surface, along with the search for extant organic materials. In addition to the elemental characterization offered by Laser Induced Breakdown Spectroscopy (LIBS), Time-Resolved Raman and Luminescence Spectroscopy (TRR/L) and visible-infrared spectroscopy (VISIR) have been added for a complete mineralogical characterization of the samples. LIBS and TRR/L techniques will be exercised from the Rover calibration targets ( $1.5 \mathrm{~m}$ range) up to $7 \mathrm{~m}$, whereas VISIR spectroscopy can be used on targets up to the horizon. A context color imagery capability is also implemented to place the analyzed samples in their geological context. A microphone allows recording aeolian phenomena, rover noises and the shock waves produced by the laser blasts on target up to 4 meters.

SuperCam consists of three units: the Body Unit built by the Los Alamos National Laboratory in the US, the Mast Unit built by a French consortium of 6 laboratories and CNES, and the Calibration Target Unit led by the University of Valladolid in Spain.
\end{abstract}

Keywords: Mars2020, Perseverance, geology, laser, spectroscopy, imaging, infrared, telescope

\section{INTRODUCTION}

On February 18, 2021, NASA will land the Perseverance rover ${ }^{1}$ on Mars's surface in Jezero Crater. Perseverance is the most sophisticated rover NASA has ever sent to Mars. It will search for signs of ancient microbial life, characterize the planet's geology and climate, collect carefully selected and documented rock and sediment samples, and pave the way for human exploration beyond the Moon. The samples will be returned to Earth by the Mars Sample Return mission in 2031.

Technical and science operations will start immediately after landing and will last at least one Mars year, starting with demonstrating Perseverance's good health and functionality for all the flight systems and the payload.

The rover carries seven science instruments to achieve Mars2020 science goals, including SuperCam, which is also called "the eye" of Perseverance. 


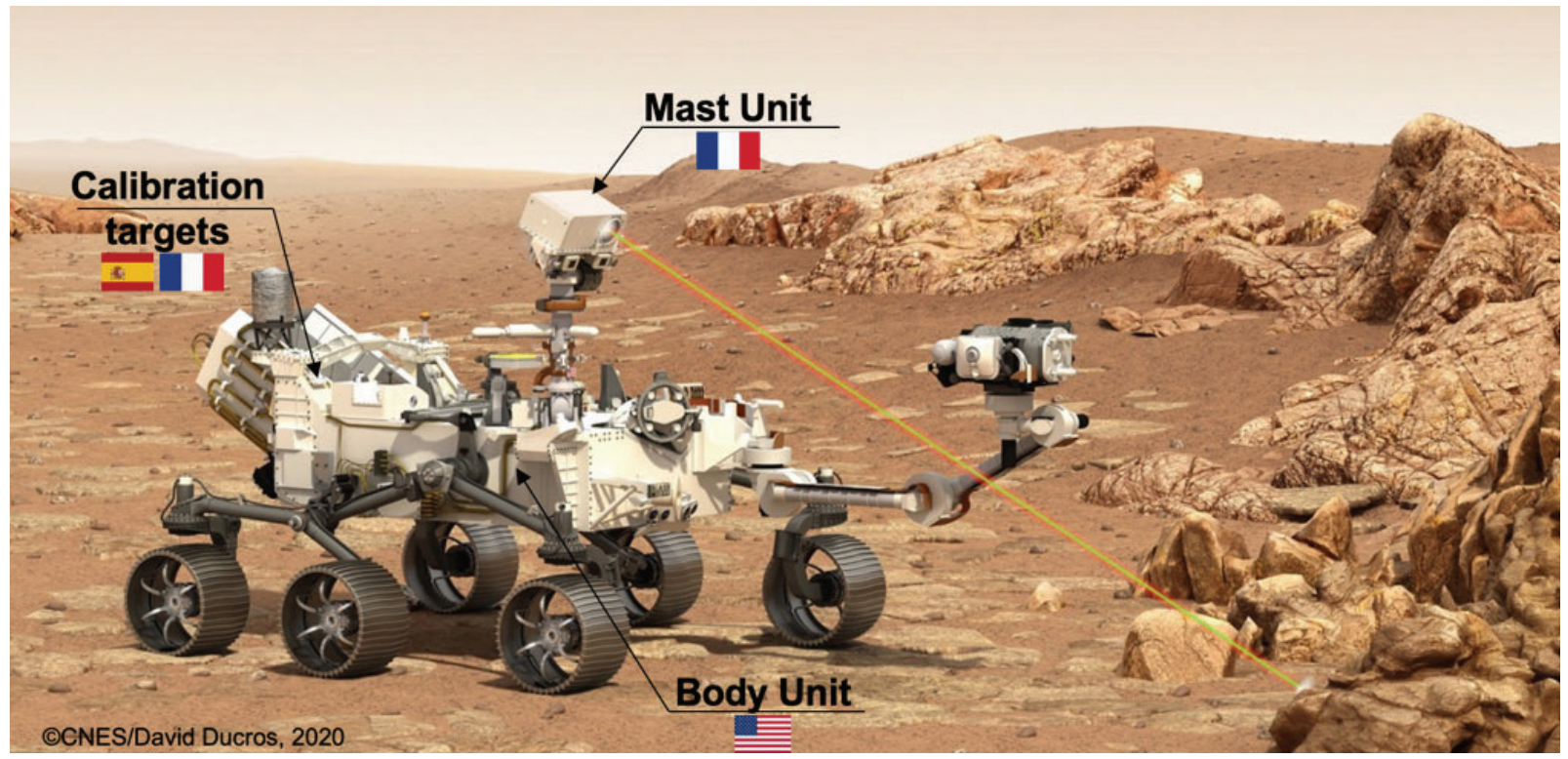

Figure 1: Perseverance Rover on Mars surface, and SuperCam instrument shooting its LIBS-Raman laser on a rock. Credit: CNES, David Ducros, 2020.

SuperCam is a suite of five co-aligned remote investigations that will work in synergy and provide analysis of rocks, regolith, and the Martian atmosphere:

- Laser Induced Breakdown Spectroscopy (LIBS): when focusing a powerful pulsed laser (1064 nm) on a rock, a plasma forms, expands and produces a broadband light emission. The plasma light spectral features are signatures of the chemical composition of the rock.

- Time-Resolved Raman and Luminescence Spectroscopy (TRR/L): a visible pulsed laser (532 nm) excites the molecules of the rock. A very small fraction of the scattering is inelastic and carries spectral information characteristic of the molecules. This Raman effect has low efficiency: on the order of 1 photon for $10^{6}$ incident or lower. Luminescence emission occurs when the molecules return from various vibrational states to their ground electronic state. The luminescence signal is typically more intense and longer-lived than the Raman signal; time resolution allows SuperCam to distinguish between them.

- Visible and Infrared Spectroscopy passive spectroscopy (VISIR): Sunlight is absorbed by molecules at frequencies driven by their vibrational frequencies. Similarly, the reflectance spectra are characteristic of the molecules composing the target. SuperCam VISIR spectroscopy will benefit from dust removal after LIBS laser shots.

- $\quad$ Remote Micro-Imaging (RMI): three-color camera with the smallest field of view of Perseverance remote sensing cameras, but the highest spatial resolution. The RMI will give the local context of millimeter-scale SuperCam observations.

- Sound recording with a microphone $\mathrm{e}^{2,3}$ (MIC): the first scientific microphone on Mars will record sounds between $100 \mathrm{~Hz}$ and $10 \mathrm{kHz}$, from natural phenomena such as winds, but also artificial rover noises, and LIBS plasma shock waves. 

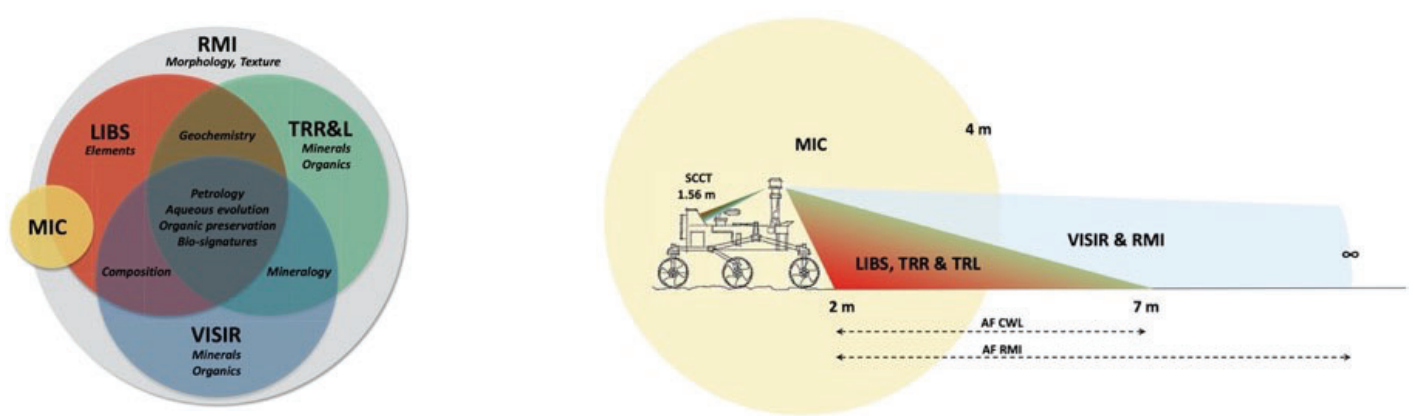

Figure 2: SuperCam investigations and their distance ranges. Credit: Maurice et al. (2021).

All techniques operate at remote distances: LIBS and TRR/L, the two investigations based on lasers, operate up to seven meters, whereas the passive techniques, VISIR and RMI, can observe up to the horizon. The microphone will be able to record the LIBS plasma shock wave up to four meters, despite the high sound absorption of $\mathrm{CO}_{2}$, the main constituent of Mars atmosphere.

The strength of SuperCam is its capability to exercise the five techniques on the same location of a target, which requires an accurate co-alignment of the different lines of sight (better than $0.35 \mathrm{mrad}$, which is half the field of view).

Table 1. SuperCam optical investigations main characteristics.

\begin{tabular}{|l|l|l|l|l|}
\hline \multicolumn{1}{|c|}{ LIBS } & \multicolumn{1}{|c|}{ TRR/L } & \multicolumn{1}{c|}{ VIS } & \multicolumn{1}{c|}{ IR } & \multicolumn{1}{c|}{ RMI } \\
\hline$\Delta \lambda: 245-853 \mathrm{~nm}$ & $\Delta \lambda: 150-4400 \mathrm{~cm}^{-1}$ & $\Delta \lambda: 400-853 \mathrm{~nm}$ & $\Delta \lambda: 1.3-2.6 \mu \mathrm{m}$ & $\Delta \lambda: 400-650 \mathrm{~nm}$ \\
$\delta \lambda: 0.15-0.65 \mathrm{~nm}$ & $\delta \lambda:<12 \mathrm{~cm}^{-1}$ & $\delta \lambda: 0.15-0.65 \mathrm{~nm}$ & $\delta \lambda:<32 \mathrm{~cm}^{-1}$ & RGB filter \\
SNR $>60$ & SNR $>20$ & SNR $>60$ & SNR $>56$ & SNR $>200$ \\
$1064 \mathrm{~nm}$ laser at $3 \mathrm{~Hz}$ & $532 \mathrm{~nm}$ laser at $10 \mathrm{~Hz}$ & Sun reflectance & Sun reflectance & Sun reflectance \\
$>12 \mathrm{~mJ}$ on target & $>9 \mathrm{~mJ}$ on target & $1 \mathrm{~m} \mathrm{to} \infty$ & $1 \mathrm{~m} \mathrm{to} \infty$ & $1 \mathrm{~m}$ to $\infty$ \\
$1.5-7 \mathrm{~m}$ distance & $1.5-7 \mathrm{~m}$ distance & $0.7 \mathrm{mrad} \mathrm{FoV}$ & $1.2 \mathrm{mrad} \mathrm{FoV}$ & $19 \mathrm{mrad} \mathrm{FoV}$ \\
Spot size $<600 \mu \mathrm{m}$ & $0.7 \mathrm{mrad} \mathrm{FoV}$ & & & Spat. res $<80 \mu \mathrm{rad}$ \\
\hline
\end{tabular}

SuperCam will scan the targets using pre-defined rasters (example of geometry: 1 x 5, 1 x 10, 3 x 3 ). All or a subset of the available techniques will be used on each point.

\section{SUPERCAM DESIGN OVERVIEW}

SuperCam is composed of three units:

- The Mast Unit ${ }^{4}(\mathrm{MU})$ is responsible for LIBS and Raman laser emissions, and collection of photons for all the techniques. Passive infrared spectra, colored images and sounds are recorded and analyzed by the Mast Unit, whereas the ultraviolet (UV), visible and near-infrared (VNIR) photons are directed to the Body Unit through a 6-meter-long optical fiber.

- The Body Unit ${ }^{5}$ (BU) splits the UV-VNIR beam in three spectral bands using an optical demultiplexer, and performs LIBS and TRR/L spectra analysis using three spectrometers. The Body Unit also manages power and data interfaces with the rover.

- The Calibration Targets ${ }^{6}$ (SCCT), located at 1.56 meters from the Mast Unit, include standards for imaging calibration, geological samples for mineral identification and chemometric calculations, or spectral references to calibrate and evaluate the health of the instrument. 


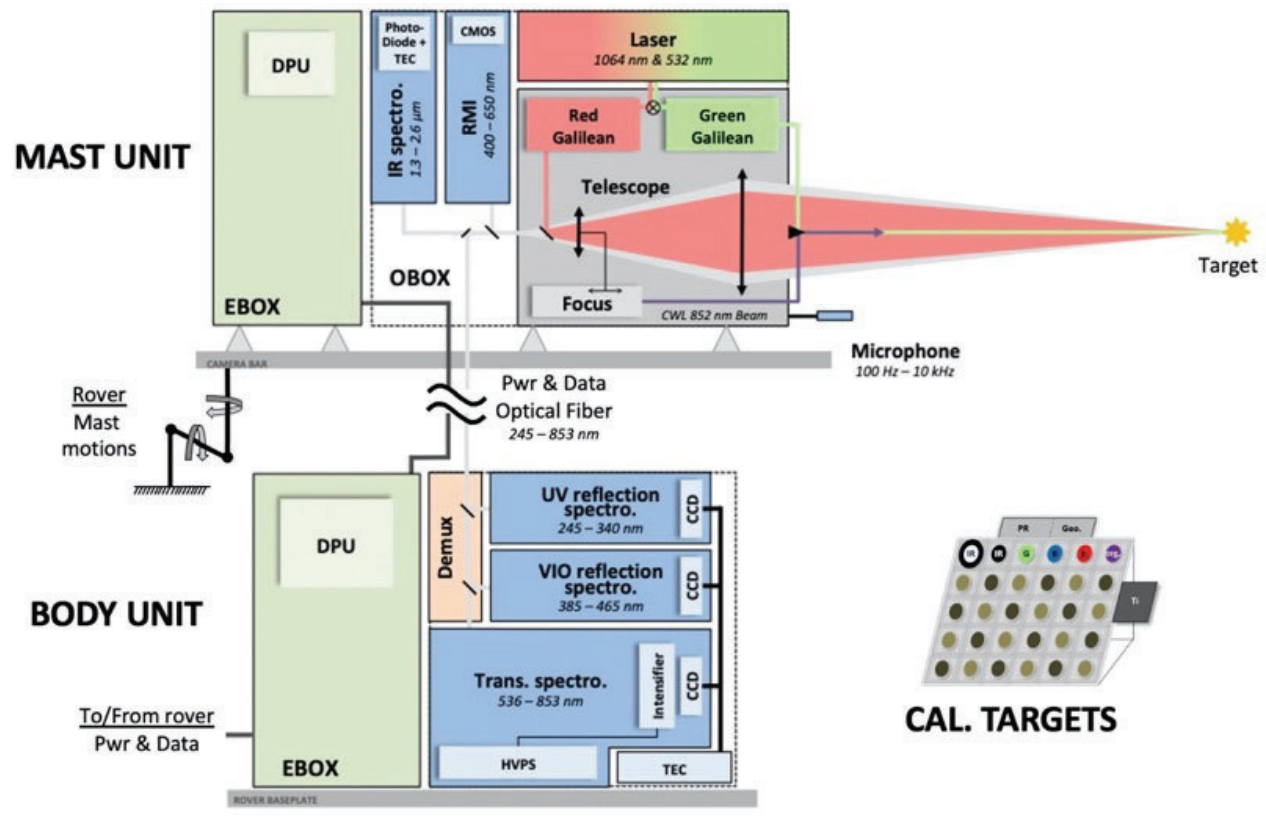

Figure 3: SuperCam block diagram. Credit: Maurice et al. (2021).
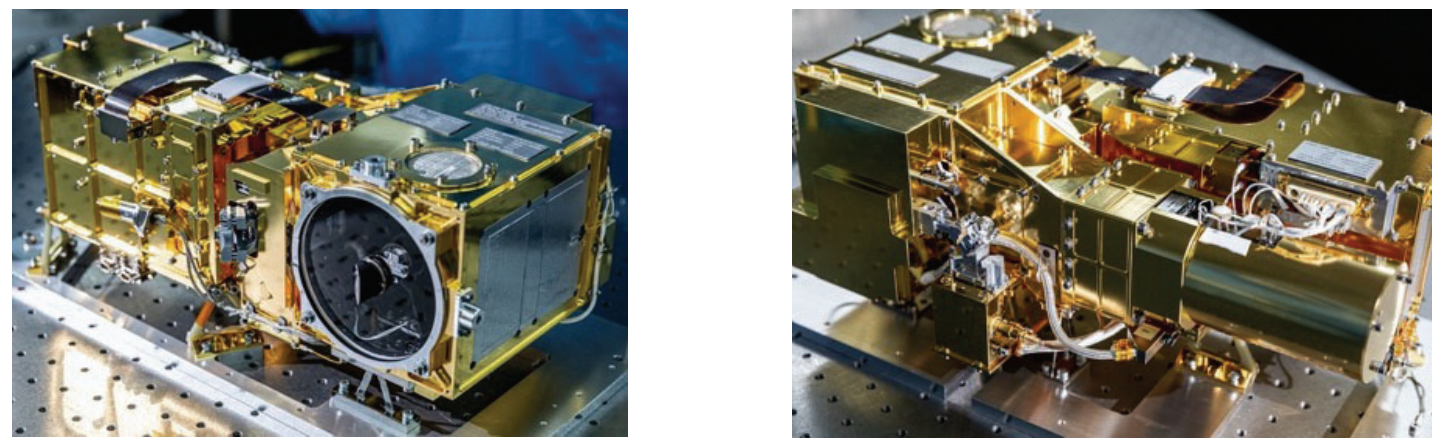

Figure 4: Mast Unit flight model pictures. Left: front view. Right: rear view. Credit: CNES, Ollier Alexander, 2019.
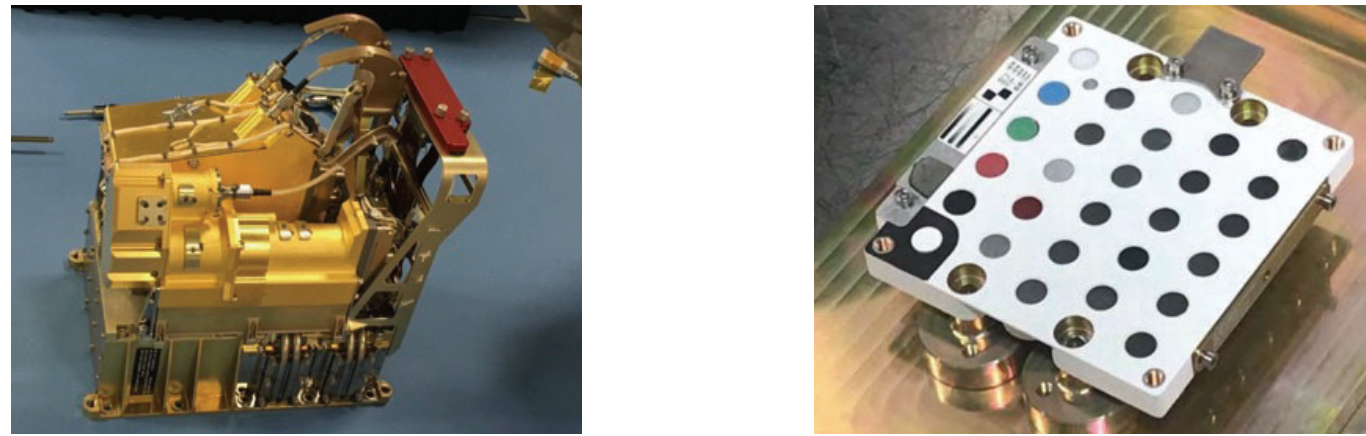

Figure 5: Body Unit flight model (left) and Calibration Target flight model (right) pictures. Credit: SuperCam, 2019.

The Mast Unit in mounted on top of the Remote Sensing Mast (RSM) of Perseverance, protected by the Remote Warm Electronics Box (RWEB), that appears in white on Figure 6 (left). The RSM can rotate $\pm 181^{\circ}$ in azimuth (AZ) and $\pm 91^{\circ}$ in elevation (EL). The Body Unit is mounted in the rover belly, protected from the harsh Martian environment. Both units are interconnected by a 6-meter optical fiber and electrical harnesses for control/command and data transfer. The Calibration targets are mounted on the rover deck. The RSM will regularly point at this assembly for instrument calibration. 

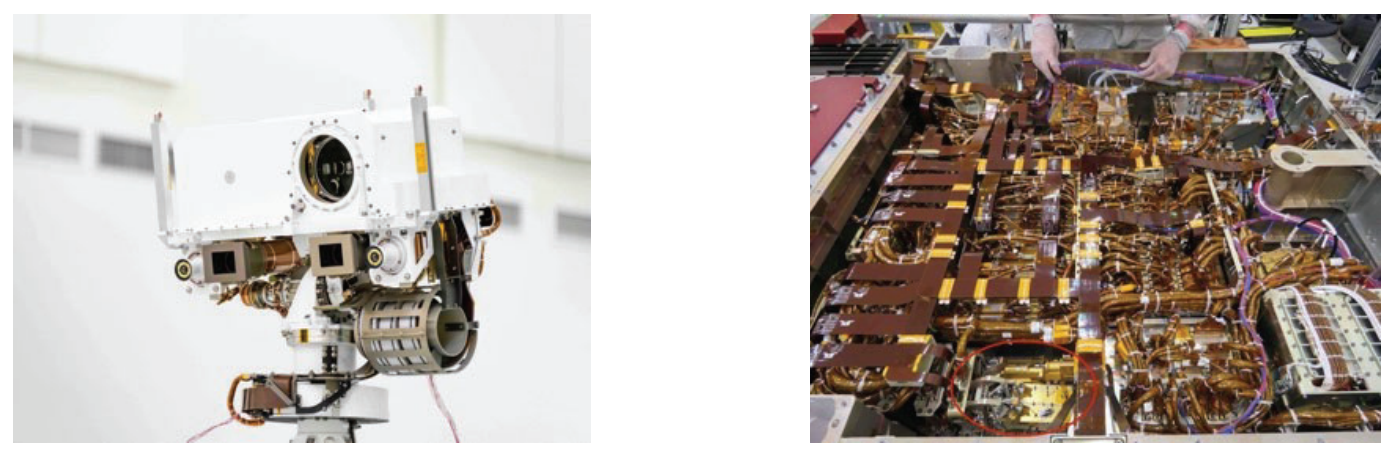

Figure 6: SuperCam accommodation on Perseverance. The Mast Unit is mounted at the top of the rover Mast (left). The Body Unit is embedded in the rover belly (right). Credit: NASA/JPL-Caltech.

Table 2. SuperCam physical properties.

\begin{tabular}{|c|c|c|c|}
\hline Parameters & Mast Unit & Body Unit & Cal. targets \\
\hline Mass & $6.1 \mathrm{~kg}$ & $4 \mathrm{~kg}$ & $0.3 \mathrm{~kg}$ \\
\hline Volume & (l) $383 \mathrm{~mm},(\mathrm{w}) 201 \mathrm{~mm}$, & (l) $221 \mathrm{~mm},(\mathrm{w}) 157 \mathrm{~mm}$, & (l) $110 \mathrm{~mm},(\mathrm{w}) 100 \mathrm{~mm}$, \\
& (h) $163 \mathrm{~mm}$ & (h) $205 \mathrm{~mm}$ & (h) $17 \mathrm{~mm}$ \\
\hline Power & $27 \mathrm{~W}$ & $43 \mathrm{~W}$ & - \\
\hline
\end{tabular}

\section{MAST UNIT OPTICAL DESIGN}

To reduce thermal gradients across the optical system, the MU consists of two units that are mechanically and thermally separated: the Electronics Box (EBOX) contains most of MU electronics; the Optical Box (OBOX) contains the $110 \mathrm{~mm}$ diameter Schmidt-Cassegrain telescope, which acts as an optical bench to carry the subassemblies: the LIBS-Raman laser, the red and green beam paths, the focusing system, the remote micro-imager (RMI), the Infrared Spectrometer (IRS) and the Microphone (MIC).

\subsection{Optical design description}

Compared to ChemCam on Curiosity, SuperCam has to accommodate a larger spectral band due to the addition of the passive IR spectroscopy investigation. Most of the MU optics have their performance specified in the [245 nm; $2600 \mathrm{~nm}$ ] spectral range, and several dichroic plates ensure the distribution of light to the different subassemblies.

LIBS laser emission. The $1064 \mathrm{~nm}$ laser beam is expanded to $1 \mathrm{~cm}$ diameter by a compact Galilean telescope and injected into the main Schmidt-Cassegrain telescope.

Raman laser emission. The $532 \mathrm{~nm}$ beam follows a different optical path. The beam is also expanded to $1 \mathrm{~cm}$ diameter by a compact Galilean telescope, but it remains collimated and is co-aligned on the telescope axis via a 2-mirror periscopic system.

Scattered light from LIBS and Raman laser shots is captured to monitor the laser energy output with a visible photodiode (laser housekeeping photodiode). This photodiode is located at the entrance of the Red Galilean expander. 

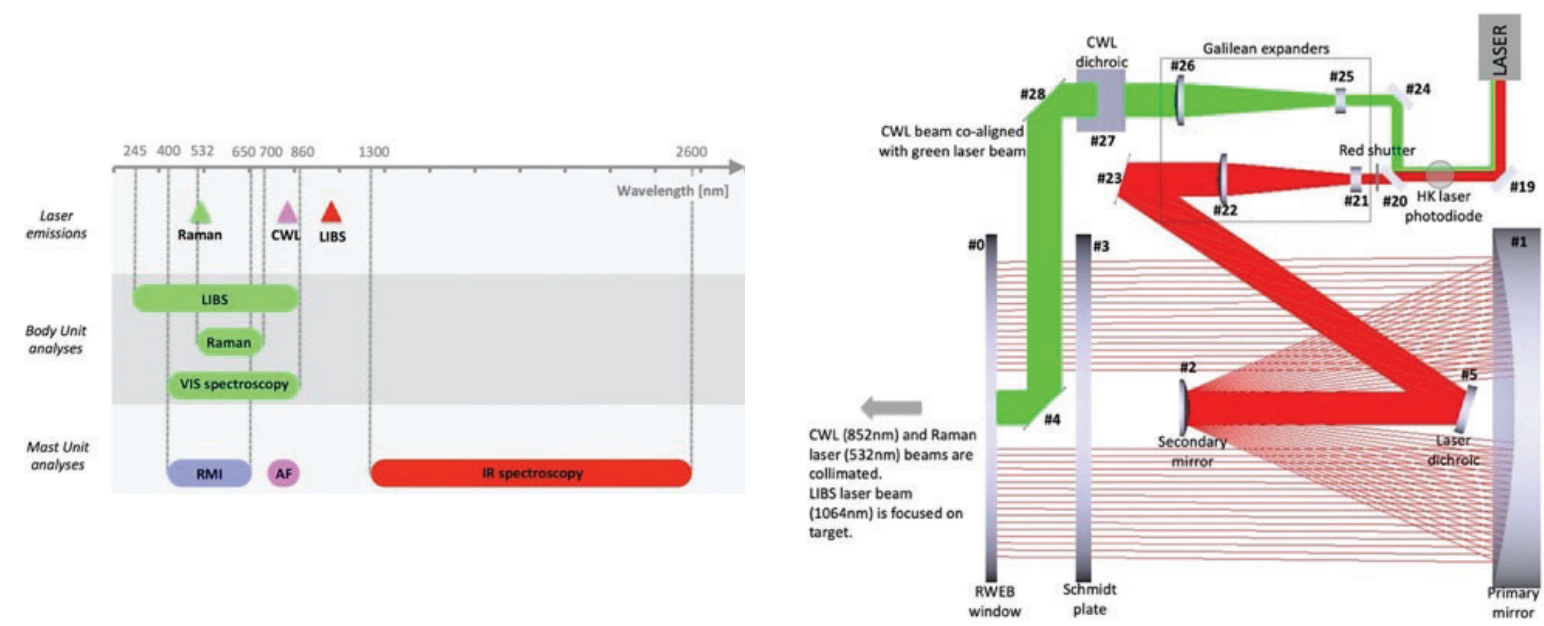

Figure 7: Mast Unit spectral range (left) and lasers emission layout (right).

Telescope. It focuses the LIBS laser beam onto the target. When the beam exits the telescope, it has the size of the primary mirror, $110 \mathrm{~mm}$ diameter. The same Schmidt-Cassegrain telescope captures light from the target. Photons traverse the laser dichroic (transparent to the 245 - $2600 \mathrm{~nm}$ range, except for the 900-1200 and 340 - $380 \mathrm{~nm}$ windows).

Objective and beamsplitter. Part of the visible light from a $20 \mathrm{mrad}$ cone around the analysis spot is focused on the RMI camera via a 3-lenses objective. The beamsplitter focuses the light below $900 \mathrm{~nm}$ into a $300 \mu \mathrm{m}$-core fiber for LIBS/Raman/VIS reflectance analyses. The last dichroic plate (\#15) acts as a notch filter working in reflection: the light is reflected in direction of the MU-BU optical fiber, except for a narrow band at $532 \mathrm{~nm}$ to prevent exciting the silica molecules of the optical fiber that would add noise on our Raman measurements.

Longer wavelengths are diverted to the IR spectrometer.

Focus. The telescope focuses on targets from $1 \mathrm{~m}$ to infinity by moving the secondary mirror with respect to the primary mirror using a translation stage mechanism. Autofocus can be achieved using two different methods: (1) Measuring the scattered light from the continuous wavelength laser (CWL) at $852 \mathrm{~nm}$ on target with a visible photodiode, (2) Finding the best contrast on the RMI images. Autofocus with CWL is used at distances lower than $7 \mathrm{~m}$ typically, and autofocus with RMI can be used at the same distances, and up to infinity. The focus requirement for LIBS and RMI is $\pm 0.4 \%$ of the distance, but is much looser for Raman and VISIR $( \pm 25 \%)$, allowing rapid scanning across uneven terrains.

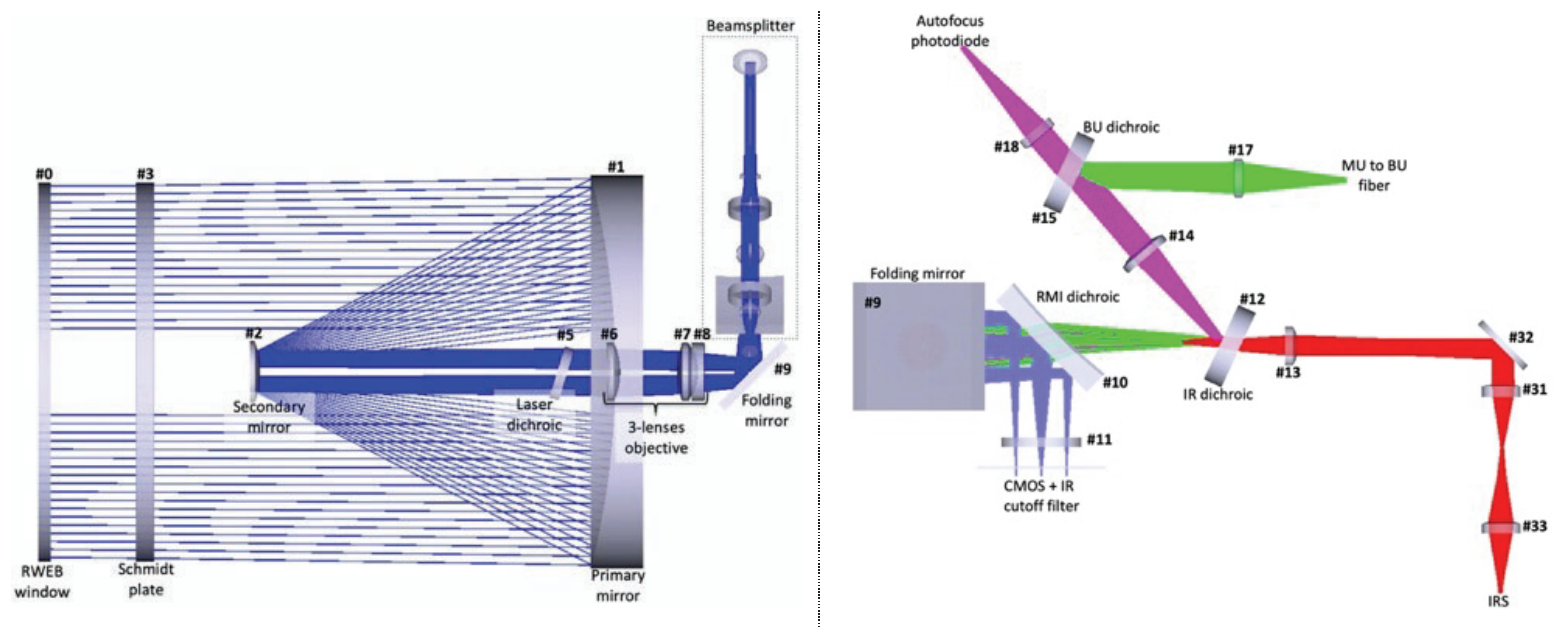

Figure 8: Mast Unit collection path. Telescope and dioptric objective (left). Beamsplitter (right). 
The MU common optical path has 31 optical elements, all custom made, except for two of them (the imager filter and the CWL aspherical collimating lens). The optics specifications result from a trade-off between performance and robustness. For example, the MU temperature will cycle every sol from $\sim-37^{\circ} \mathrm{C}$ to $\sim-10^{\circ} \mathrm{C}$, causing stress in the optical coatings if they are too thick. Caution was taken to limit the number of optical layers $(<100)$ for the most complex dichroic plates, and all the coated optics were tested under thermal cycling $\left(1500\right.$ times $-40^{\circ} \mathrm{C}$ to $\left.+35^{\circ} \mathrm{C}, 5^{\circ} \mathrm{C} / \mathrm{min}\right)$.

The optics located at the laser output see high fluence $\left(0.7 \mathrm{~J} / \mathrm{cm}^{2}\right.$ at $1064 \mathrm{~nm}$ and $0.4 \mathrm{~J} / \mathrm{cm}^{2}$ at $\left.532 \mathrm{~nm}\right)$, and their coating was tested under these laser fluxes. Dedicated test campaigns were also run to assess the risk of laser induced contamination, and specify the maximum acceptable level for molecular contamination.

Apart from the optics procurement, the two main challenges for the MU optical design were:

- The LIBS focusing: LIBS spectroscopy requires to reach a high irradiance on target $\left(>1 \mathrm{GW} / \mathrm{cm}^{2}\right)$ to $\mathrm{create}^{\mathrm{a}}$ plasma. This is achieved by optimizing the laser energy on target, but first and foremost by focusing the laser beam on a very small spot. Some performance loss was discovered late in the development at cold temperatures $\left(<-30^{\circ} \mathrm{C}\right)$. The root cause was that a nickel layer below the primary mirror coating, coupled its light-weight shape, was degrading the surface wavefront error at cold. The correction was to manufacture (in a very short amount of time!) a new primary mirror without nickel layer.

- The Raman laser alignment: on the contrary to LIBS, the Raman laser beam does not benefit from the "natural" co-alignment with the telescope optical axis, and is co-aligned on MU line of sight through a periscopic system. The second mirror of this periscope (element \#4 on Figure 7) is mounted at the center of the telescope Schmidt plate, and slightly rotates when the telescope structure is varying with temperature. The consequence is a small motion of the Raman laser beam along the vertical axis, combined with hysteresis due to the frictions at the Schmidt plate/optical bench interface. Design adjustments were made to minimize friction. Also, the alignment strategy consisted in biasing the alignment at room temperature in order to be optimized at $-20^{\circ} \mathrm{C}$ in the middle of the performance temperature range.

\subsection{LIBS-Raman laser}

The laser ${ }^{7}$ is the heart of the LIBS and Raman experiments. The laser cavity is based on a Nd:YAG crystal to provide the red line at $1064 \mathrm{~nm}$. The Nd:YAG crystal is pumped by a multi-color laser diodes stack emitting in the $795-805 \mathrm{~nm}$ spectral band to cope with the narrow spectral acceptance of the Nd:YAG crystal, and its variations with temperature. At the cavity output, a second harmonic generator (KTP crystal) multiplies the beam frequency by two to provide the line at $532 \mathrm{~nm}$. The phase matching is achieved using a Pockels cell to rotate the beam polarization. The laser needs a pumping current up to $155 \mathrm{~A}$ and high voltage of $1200 \mathrm{~V}$ to reach its full performance. The LIBS energy is higher than $24 \mathrm{~mJ}$, the Raman energy is higher than $12 \mathrm{~mJ}$, with a pulse duration of $4 \mathrm{~ns}$. The laser can be used with repetition rate in the $1-10$ $\mathrm{Hz}$ range. The near field beam has a rectangular shape of dimensions $2.1 \times 2.3 \mathrm{~mm}^{2}$ at $1 / \mathrm{e}^{2}$ at room temperature. The M2 factor is 1.3, and allows a good focus of the LIBS laser beam on target. The laser cavity is sealed (dry air, 1 bar pressure inside), and interfaced on the OBOX through a Titanium bracket.

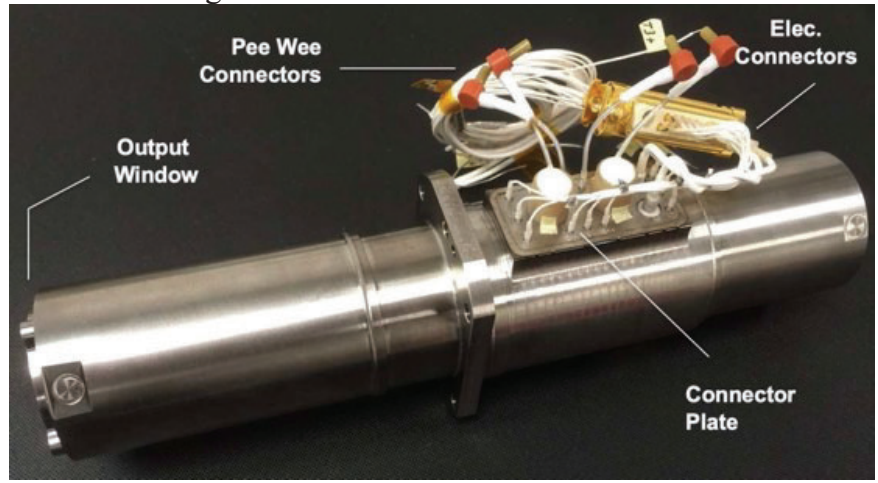

Figure 9: LIBS-Raman laser. Credit: Thales-LAS. 


\subsection{Remote Micro-Imager (RMI)}

The RMI ${ }^{8}$ is based on a CMV4000 CMOS sensor $(2048$ x 2048 pixels of $5.5 \mu \mathrm{m})$ with a Bayer filter, embedded in a cube containing readout and control electronics. A NIR cut-off filter is placed in front of the cube to retrieve the colors. The useful field of view is $18.8 \mathrm{mrad}$ in diameter, with a spatial resolution better $80 \mu \mathrm{rad}$ over $10 \mathrm{mrad}$ field of view in diameter, and a scale of $10 \mu \mathrm{rad}$ per pixel. The RMI has an auto-exposure capability, and a High Dynamic mode to improve contrast and signal-to-noise ratio in the darkest zones of the image.
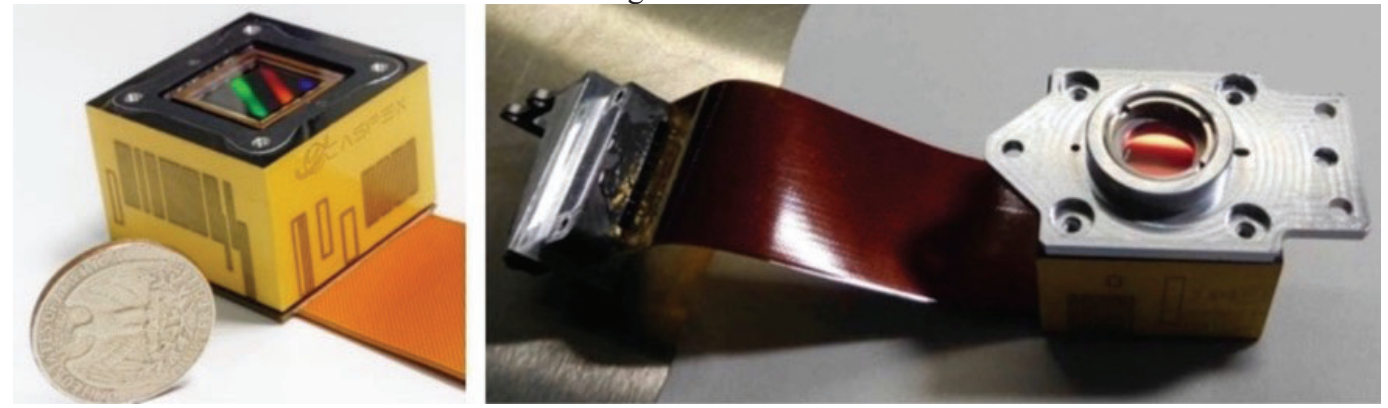

Figure 10: Remote Micro-Imager. The 3D+ cube (left) and the cube with the NIR cutoff assembly (right). Credit: SuperCam.

\subsection{InfraRed Spectrometer (IRS)}

The IR spectrometer ${ }^{9,10}$ is an independent device, linked to the telescope objective by a periscopic system. The entrance of the IRbox is a pinhole ( $400 \mu \mathrm{m}$ in diameter, 0.18 numerical aperture). The spectral selection is done by an Acousto-Optical Tunable Filter (AOTF), manufactured by Gooch \& Housego, which allows a very compact and robust spectrometer with no moving parts. A radio-frequency signal drives a transducer attached to the side of the AOTF. For each frequency of the piezo, a single wave number is selected and scattered by $\pm 6.5^{\circ}$ ( 2 polarizations). The main beam is rejected, while both polarizations are registered by two $\mathrm{HgCdTe}(\mathrm{MCT})$ photodiodes (cold redundancy), from Teledyne Judson Technologies. These MCT photodiodes are packaged with a triple-stage TEC. The detector TEC can lower the photodiode temperature to $70^{\circ} \mathrm{C}$ below that of the spectrometer. Spectra up to 256 spectels can be registered in the $1.3-2.6 \mu \mathrm{m}$ spectral band, with a spectral resolution better than $32 \mathrm{~cm}^{-1}$ and signal-to-noise ratio of 56 or better.

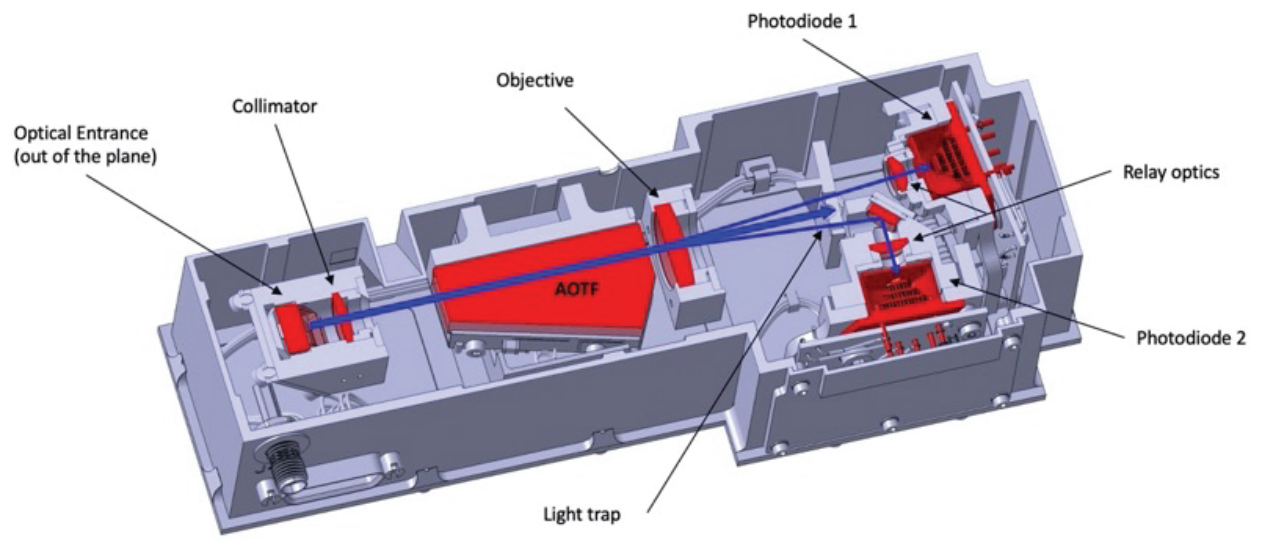

Figure 11: InfraRed Spectrometer (IRS) CAD view. Credit: SuperCam. 


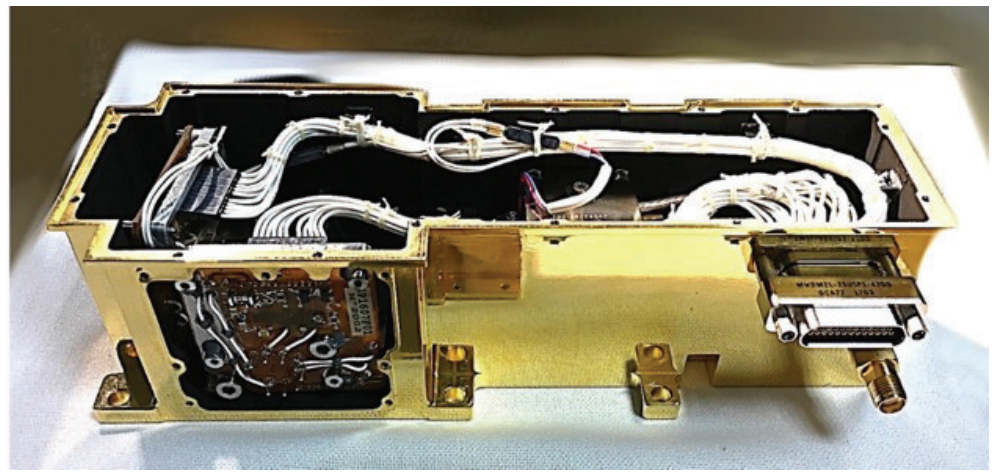

Figure 12: InfraRed Spectrometer (IRS) flight model picture. Credit: SuperCam.

In order to correct the data, the instrument function shall be carefully calibrated ${ }^{11}$. This required a 10 -day test campaign just before we delivered the MU to the Jet Propulsion Laboratory (JPL) in California.

\section{BODY UNIT OPTICAL DESIGN}

The BU is composed of the optical demultiplexer (demux) that receives light from the MU through the 6-meter optical fiber, three spectrometers working from the UV to the VNIR, and an electronics box (EBOX) to drive the BU subsystems and manage the interface with MU and the rover. The three spectrometers are used for LIBS. TRR/L signals are recorded by the visible-near infrared (VNIR) spectrometer, also called transmission spectrometer. Visible passive spectroscopy uses the VIO and VNIR spectrometers.

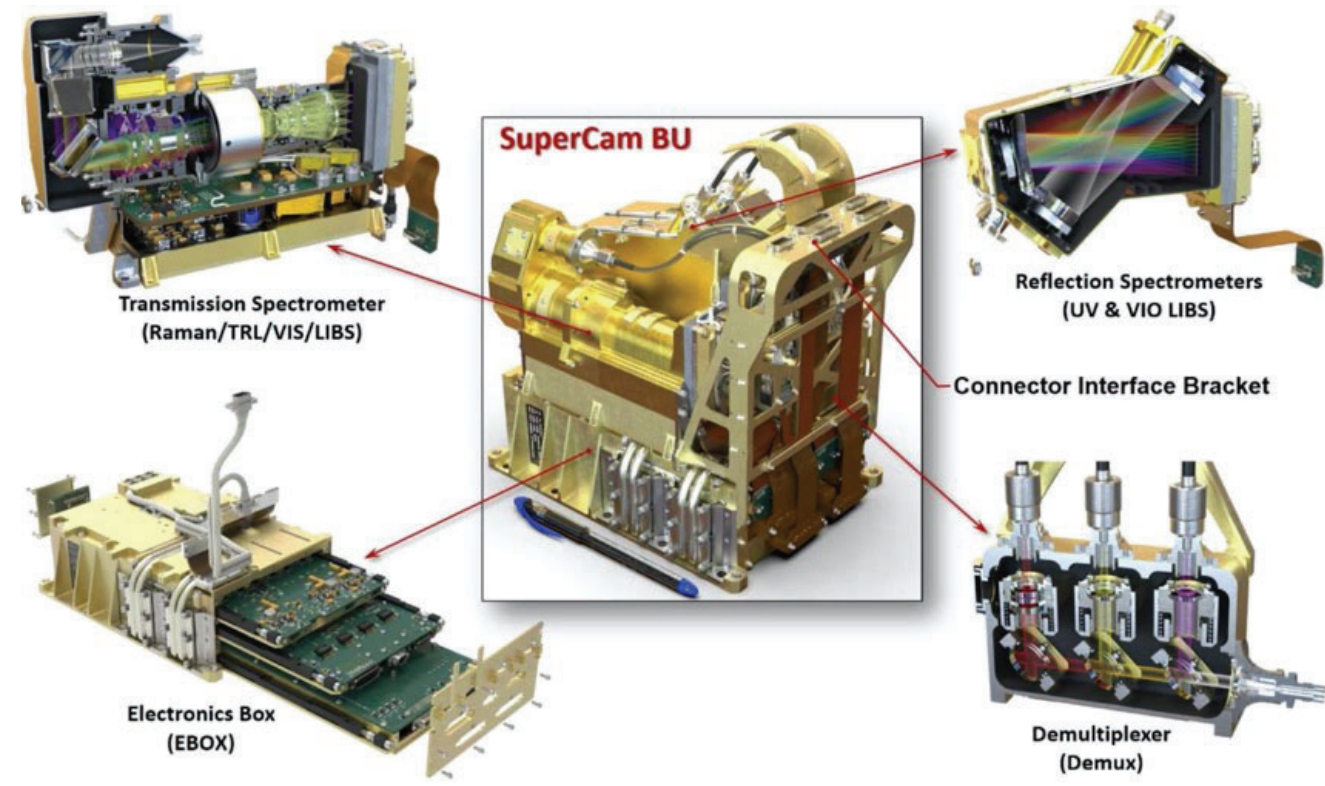

Figure 13: Body Unit design overview. Credit: SuperCam.

\subsection{Demux}

The demux function is to split the incoming light collected by the MU in three spectral bands to pass to the three spectrometers:

- UV: $245-342 \mathrm{~nm}$ 
- $\quad$ VIO: $382-468 \mathrm{~nm}$

- $\quad$ VNIR: $535-853 \mathrm{~nm}$

The optical entrance is an AVIM connector. The beam is collimated and spectral bands are selected by a series of two dichroic plates. The VNIR path also includes a long-pass filter with a sharp transition at $534 \mathrm{~nm}$ in order to block the remaining $532 \mathrm{~nm}$ laser light from reaching the spectrometer optics. The beams are then focused on the demux outputs connected to fiber bundles to reorganize the beam circular shapes in linear shapes that fit the spectrometers entrance slit. The bundles comprise 19 fibers in a circular array at the input, and a linear array at the exit. The fibers, each with a $50 \mu \mathrm{m}$ core, a map such that the center fiber in the circular array is at the center of the line, the six fibers surrounding the center are placed three on either side, and so forth.
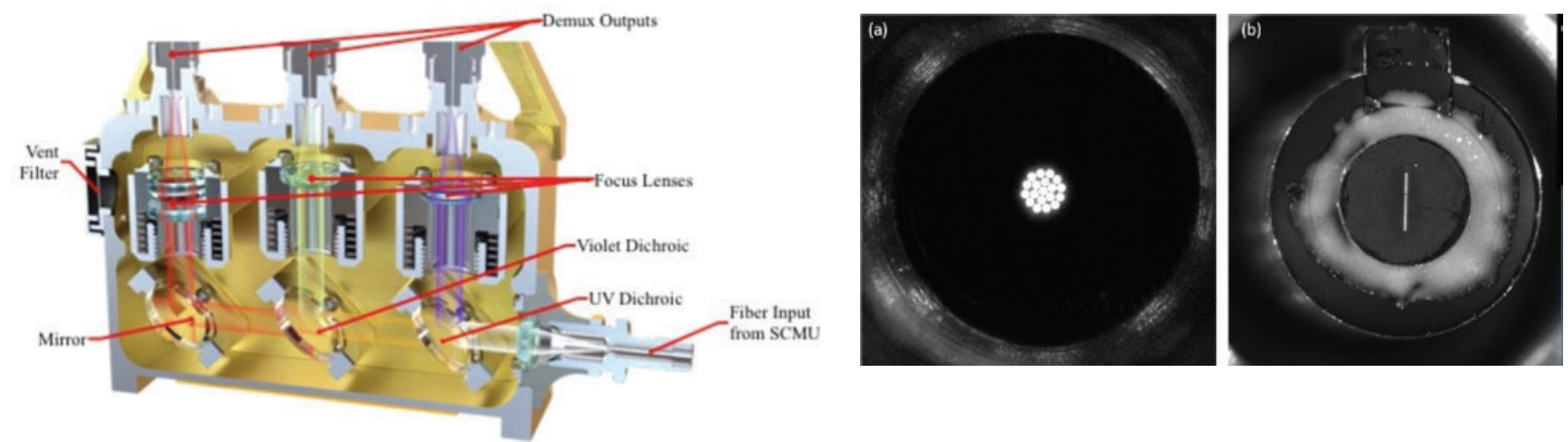

Figure 14: Optical demultiplexer (demux) on the left, and an example of fiber bundle on the right ((a) shows the demux output and (b) the spectrometer entrance). Credit: SuperCam

\subsection{UV \& VIO spectrometers}

The UV and VIO spectrometers have a classic Czerny-Turner design, and benefit from a high heritage from ChemCam. They are equipped with 2400 lines per mm reflection gratings, and 42-10 CCDs from E2V (2048 x 515 pixels, $13.5 \mu \mathrm{m}$ pitch).

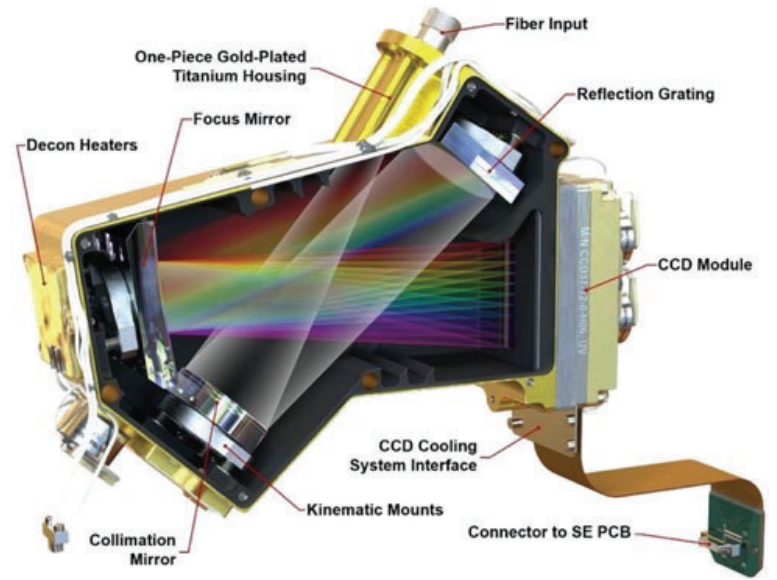

Figure 15: UV \& VIO spectrometers. Credit: SuperCam.

\subsection{Transmission spectrometer}

The transmission spectrometer is a new development for SuperCam to be able to detect the very weak Raman signals. The key features are the high throughput, signal intensification and time-gating. 
The entrance beam is collimated and split in two spectral bands before reaching a pair of volume-phase (VPH) gratings. One of the grating assemblies contains a combination of two gratings written in the same substrate with a $1.8^{0}$ separation angle between them. The 3 spectral orders are then focused on the intensifier. After intensification, a set of relay lenses reimages the beam on the CCD (same reference than the reflection spectrometers).

The image intensifier is the MX-10130 from ITT Exelis, biased and gated using a newly-designed high voltage power supply (HVPS) located below the opto-mechanical part of the spectrometer. The gain of the intensifier can be adjusted by choosing the value of high voltage of the microchannel plate. The timing of the high voltage is controlled in order to timegate the signal. Time-gating is synchronized with the CCD readout. These parameters shall be adapted to the technique as the LIBS produces a signal far brighter than Raman spectroscopy. Whereas LIBS and Raman signals are in the range of nanoseconds, fluorescence is optimal after microseconds.

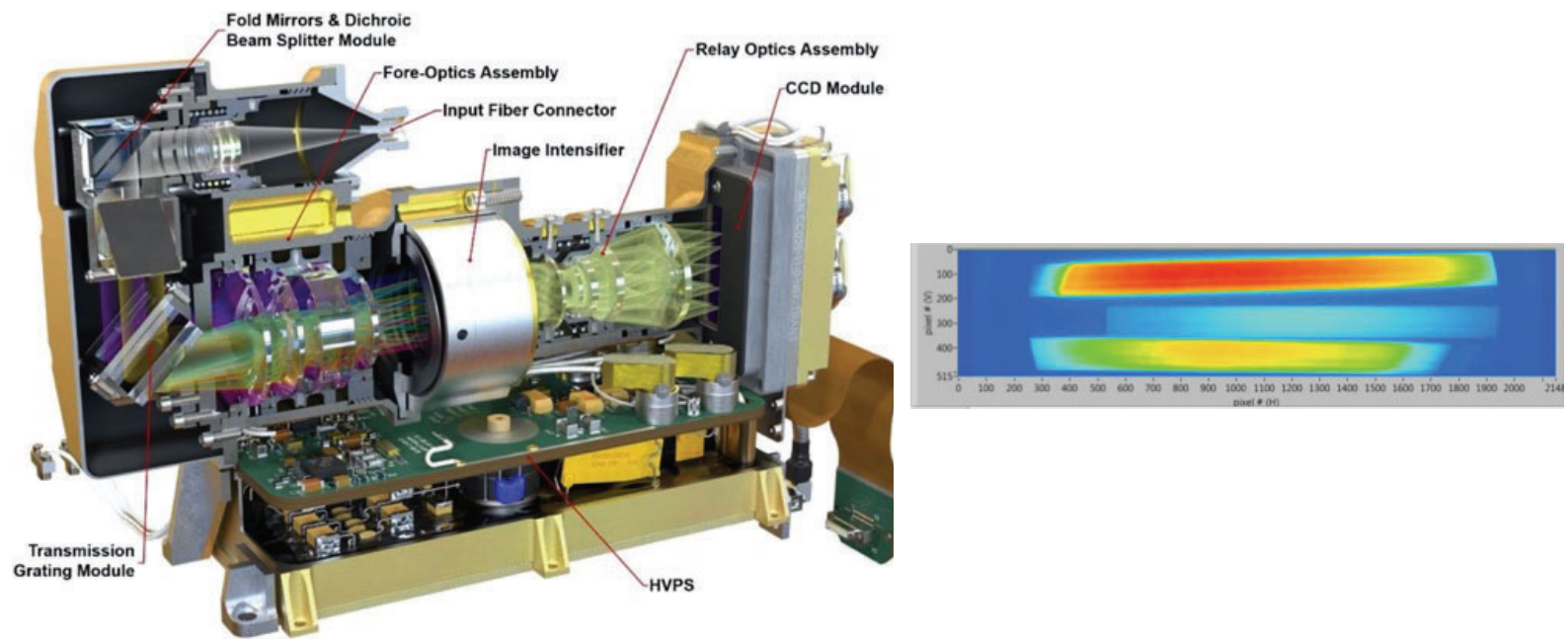

Figure 16: Visible and near-infrared transmission spectrometer (left). Three grating orders projected on the CCD (right). Credit: SuperCam.

\section{PERFORMANCE}

MU and BU were qualified and tested separately in France and at Los Alamos National Laboratory (US) to verify the functional and performance requirements at subunits level. The instrument performances (coupling of MU and BU) were assessed on Perseverance rover at JPL during a 6-month test campaign in July-December 2019.

\subsection{Mast Unit}

Telescope focus. Both autofocus modes (CWL and RMI) were extensively tested in thermal environment and using rocks of different structures and albedo. Robustness and repeatability were verified. Optical focus offsets between the different techniques were characterized; they will be automatically corrected on-board.

The CWL autofocus is optimized when sun illumination is weak or the target is in shadow. The scan range is large and thus tolerant to uncertainty of the seed distance. RMI autofocus takes more time to run, but is better suited to longer target distances. The scan is very small and requires a good knowledge of the seed distance, compatible with the rover capability. 


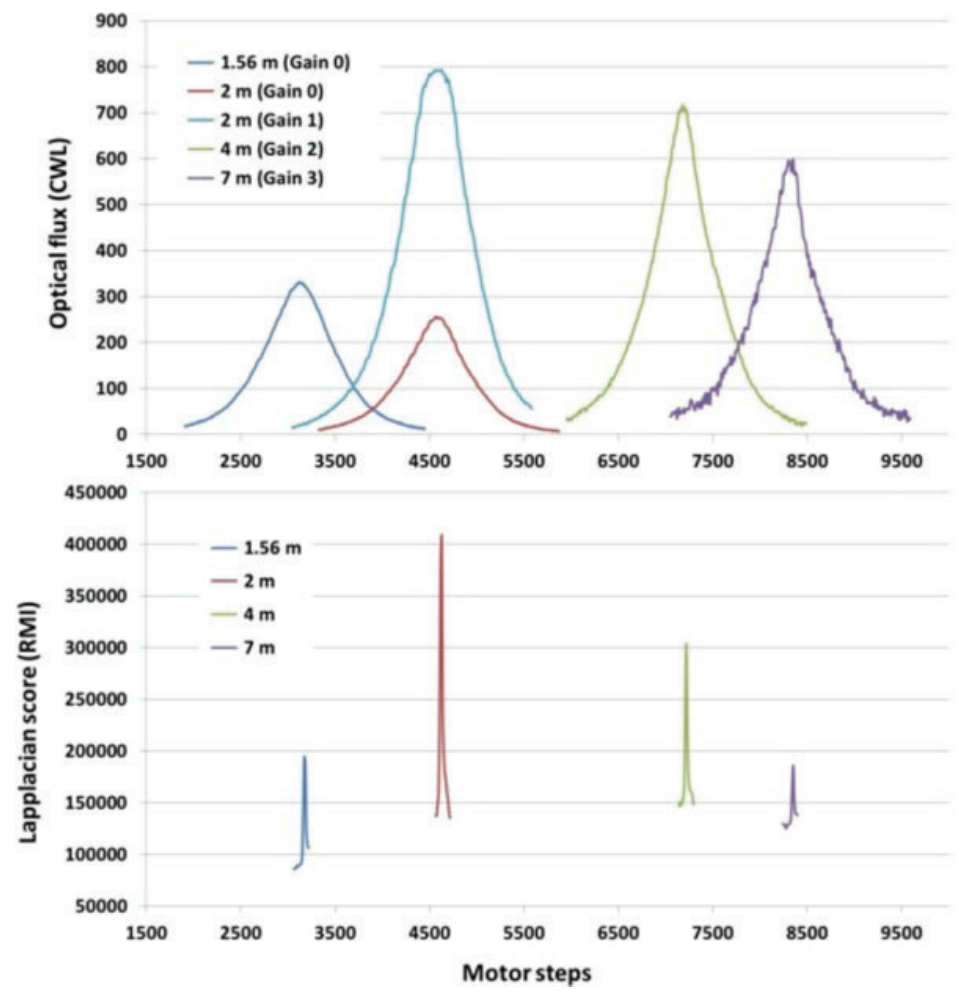

Figure 17: Autofocus curves for the same target at four distances. (Top) CWL autofocus curves. At $2 \mathrm{~m}$, the measurement is repeated and ended up with a different gain, but the same focus to within 1 motor step. (Bottom) RMI autofocus curves. Credit: Maurice et al. (2021).

LIBS laser emission. The telescope equipped with the newly-designed primary mirror (without nickel layer) is almost diffraction-limited down to $-40^{\circ} \mathrm{C}$. Combined with more than $12 \mathrm{~mJ}$ deposited on the target, the irradiance is well above the minimum requirement of $1 \mathrm{GW} / \mathrm{cm}^{2}$. At short distances, it is recommended to lower the laser pumping current in order to avoid the spectrometers saturation.
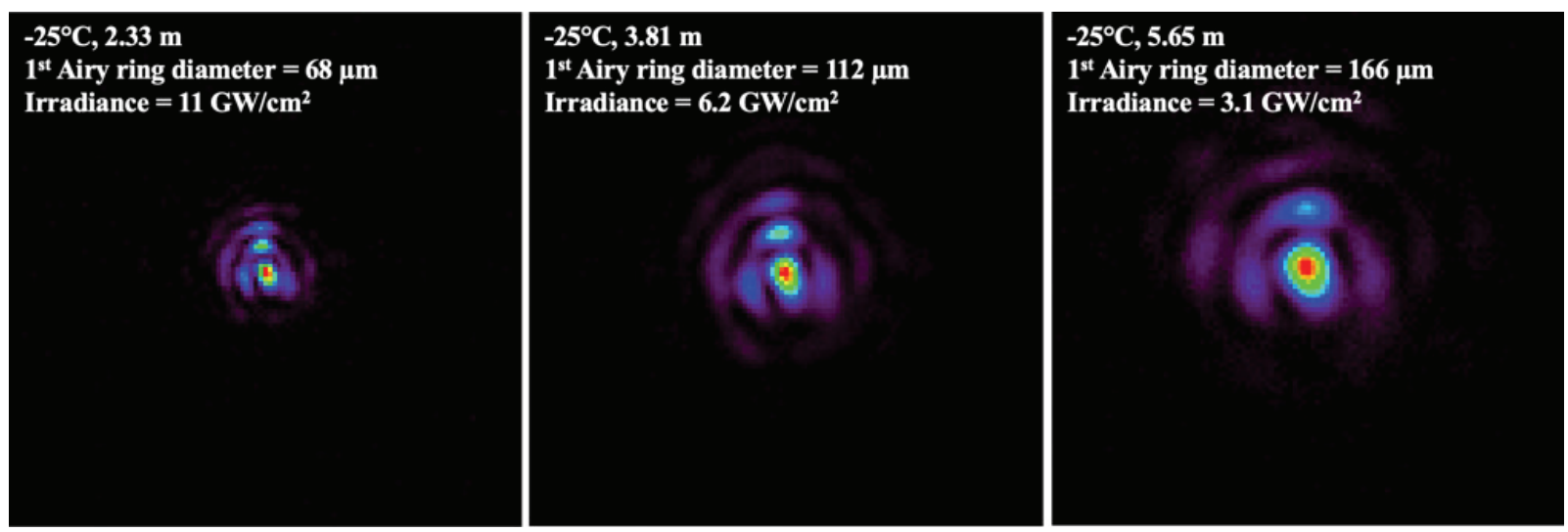

Figure 18: LIBS point spread function at different distances and $\mathrm{T}=-25^{\circ} \mathrm{C}$. The theoretical $1^{\text {st }}$ Airy ring is indicated, as well as the irradiance on target. The minimum requirement is $1 \mathrm{GW} / \mathrm{cm}^{2}$.

Raman laser emission. The irradiance on target varies with temperature and distance mostly due to the laser beam divergence, but also to the energy provided by the laser. Most of the time the irradiance will be in the range of $15-30$ $\mathrm{kW} / \mathrm{mm}^{2}$ (where the science team expects $30 \mathrm{~kW} / \mathrm{mm}^{2}$ ). It can drop to $10 \mathrm{~kW} / \mathrm{mm}^{2}$ at the extreme case of 7 -meter range 
and OBOX temperatures lower than $-31^{\circ} \mathrm{C}$. Firing the Raman laser with longer bursts (up to 200 shots) and co-adding spectra on the CCD sensor will help increasing the signal to noise ratio.
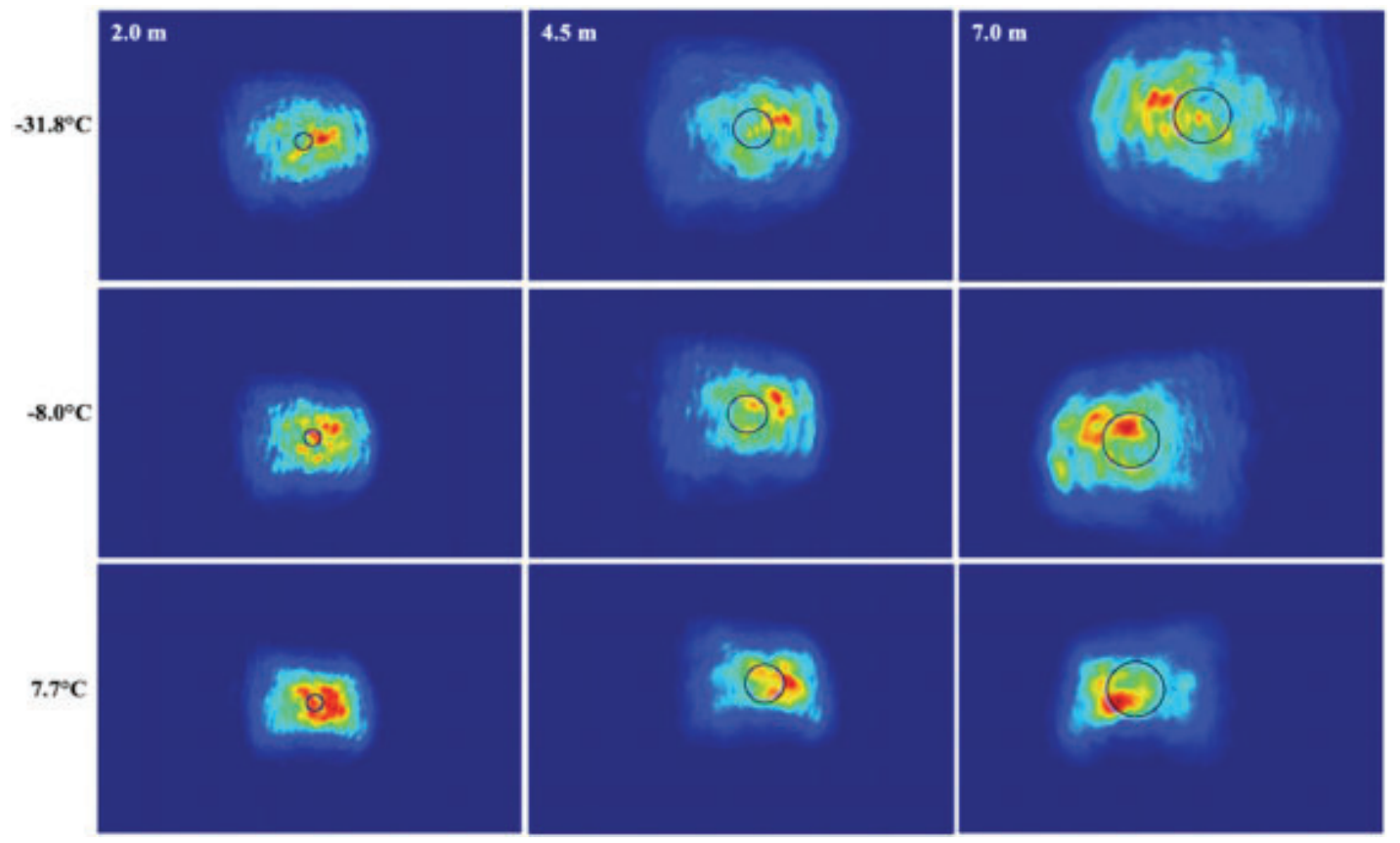

Figure 19: Alignment of the Raman laser (in green) with respect to the telescope line of sight (black circle that represents the

$0.7 \mathrm{mrad}$ field of view) at different temperatures and distances, and during a simulated Mars cooling cycle.

Substantial effort was invested to optimize the Raman laser beam alignment on the telescope line of sight. This includes late design changes, careful alignment procedure, and verification tests at each step of the integration and environmental tests. The last verification was made at the JPL Spacecraft Assembly Facility in December 2019, just before Perseverance left Pasadena for integration to the rocket stack.

Imaging. The RMI field of view $(18.8 \mathrm{mrad})$ and flat field were accurately characterized for correction on-ground. The spatial resolution, driven by the telescope optical quality, is defined as the frequency at which the modulation transfer function is equal to $20 \%$. It was measured as a function of temperature, distance and position in the field of view.
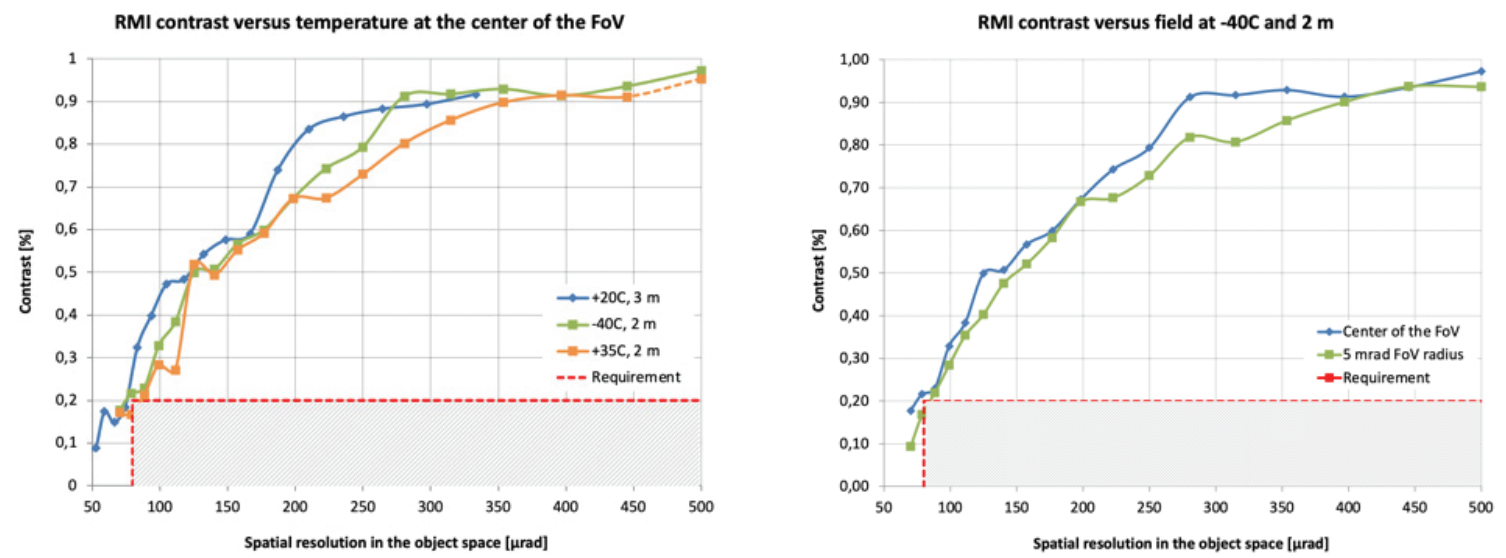

Figure 20: RMI resolution. (Left) Contrast always at the center of the field of view for different temperatures. (Right) Contrast at the center of the field of view and at $5 \mathrm{mrad}$ off the center. The requirement is to be above the dotted line in red. Credit: Maurice et al. (2021). 
The capability to record images from short distances (even lower than $1.56 \mathrm{~m}$ corresponding to the SuperCam calibration targets on the rover deck), and up to infinity was demonstrated.
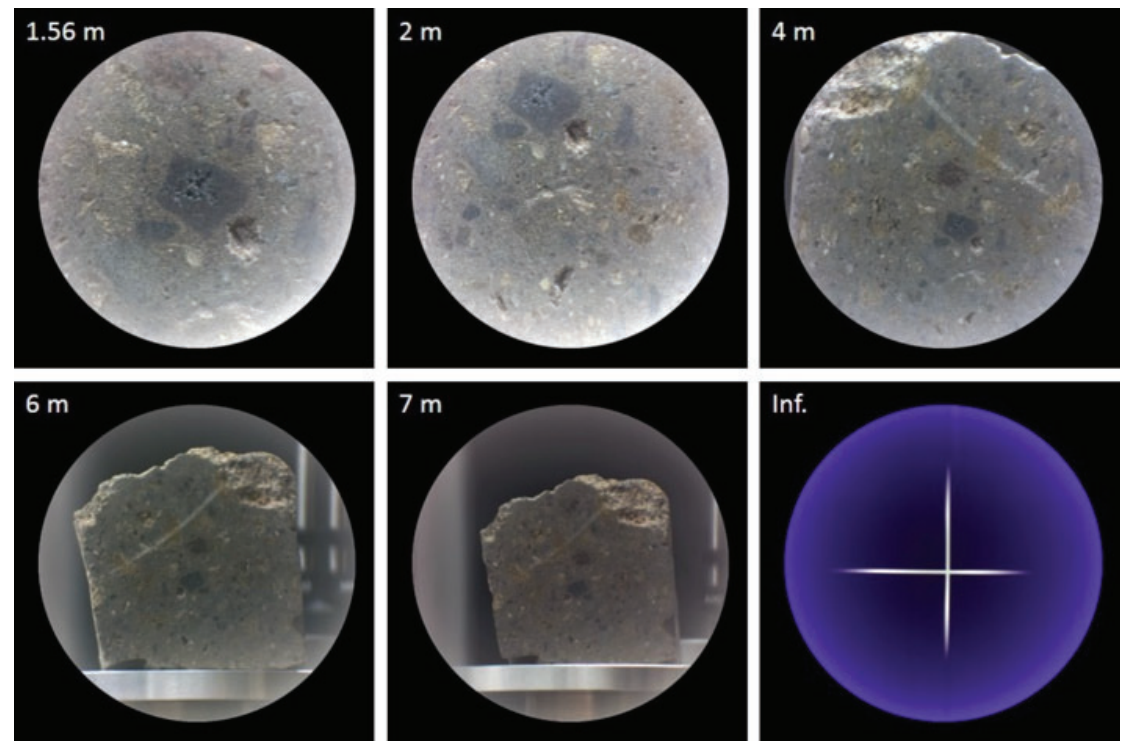

Figure 21: Series of RMI images acquired at $-40^{\circ} \mathrm{C}$ during the MU thermal tests. The same rock is placed at different distances. Its size is $\sim 7.3 \mathrm{~cm}(\mathrm{~L}) \mathrm{x} \sim 7.2 \mathrm{~cm}(\mathrm{H})$. The dark crystal at the center of the first image measures $\sim 8 \mathrm{~mm}(\mathrm{~L})$ by $\sim 7 \mathrm{~mm}(\mathrm{H})$. LIBS pits can be seen. The last is an image of a collimator reticule that mimics a target at infinity. Credit: SuperCam.

Infrared spectrometer. The IRS was fully characterized at subsystem level at LESIA facilities (Meudon, France). Spectral and radiometric performance were assessed in thermal environment for both photodiodes.

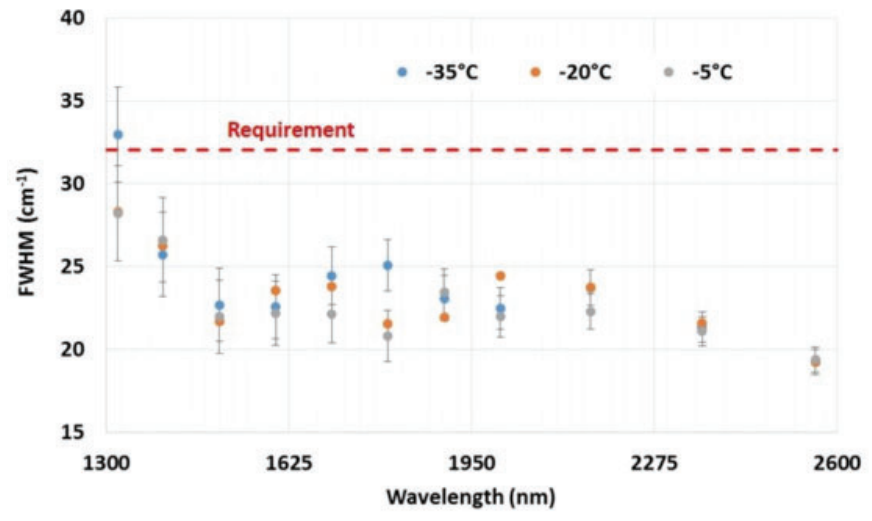

Figure 22: The IRS spectral resolution (nominal photodiode) measured for three temperatures of the spectrometer. The requirement is to be below the red dotted line at $32 \mathrm{~cm}^{-1}$. Credit: Maurice et al. (2021).

The measurements were used to feed the radiometric model and predict the signal to noise expected on Mars, depending on the thermal environment properties and hour of day. 


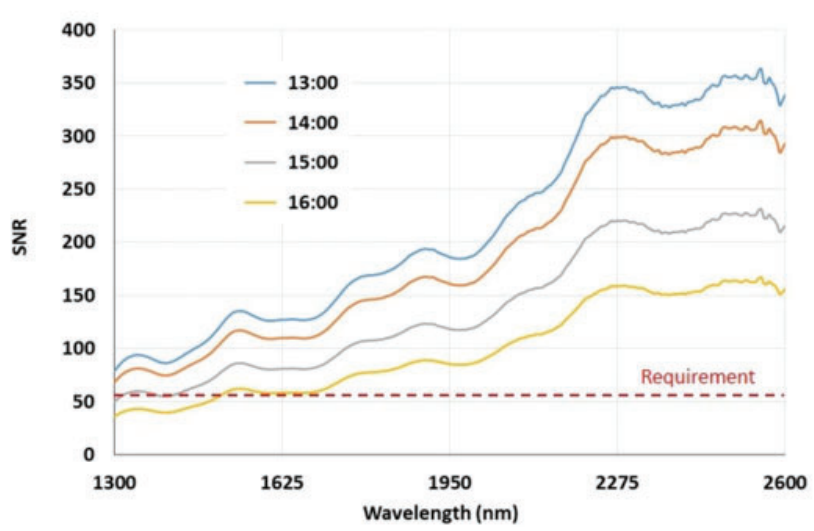

Figure 23: Signal-to-noise simulations for the IRS, as a function of local time (HH:MM), for a thermal environment close to Jezero crater and a start of the instrument at 13:00. This is a worst hot case. Four SNR simulations are shown, as the instrument is warming up, for 86 spectels acquired in $80 \mathrm{~s}$ (signal and dark). The requirement is to be above the dotted line at SNR $=56$. Credit: Maurice et al. (2021).

Before leaving France, MU underwent a calibration campaign ${ }^{11}$ for the IR channel, to measure the radiometric and linearity responses of the instrument over its nominal range of operations, in terms of instrument detector temperatures and spectral range. The derived instrument transfer function will be used to calculate the expected instrumental signal-to-noise ratio for typical observation scenarios of mineral mixtures expected to be found in Jezero crater, and ultimately to retrieve the spectral properties of the regions of interest observed by the rover.

\subsection{Body Unit}

Spectral characteristics. Spectral ranges, spectral resolution, and spectral registration (wavelength versus pixels) were characterized over BU expected temperature range on Mars. Spectral resolution requirement is easily met and remains acceptable over a large temperature range from $-40^{\circ} \mathrm{C}$ to $+35^{\circ} \mathrm{C}$. Spectral registration also varies with temperature: a drift of $\sim 1$ pixel over $35^{\circ} \mathrm{C}$ was observed, whereas 0.2 pixels stability is required. Data could be corrected on-ground thanks to regular spectral calibration using LIBS technique on the SuperCam Titanium target.

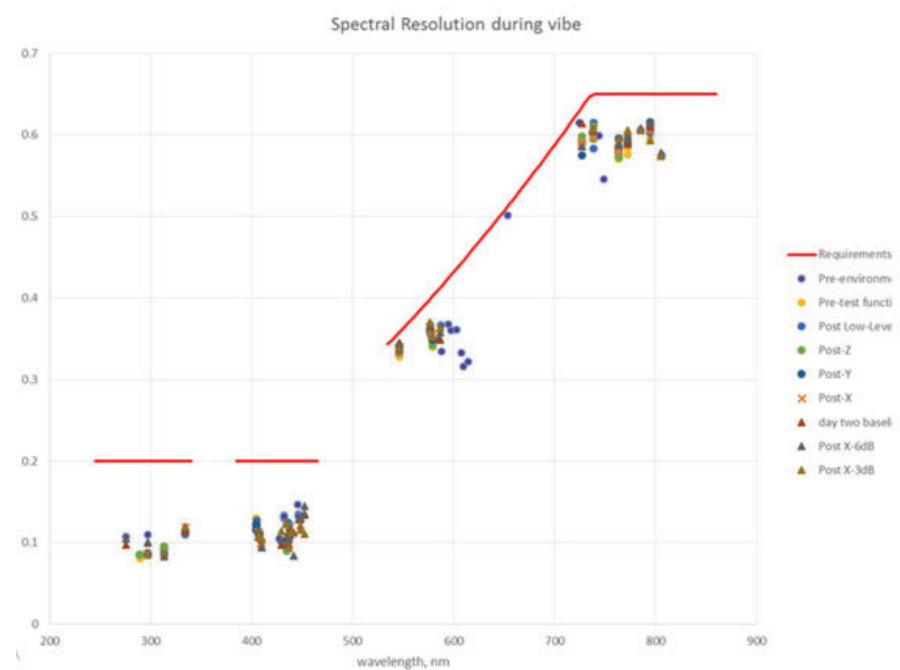

Figure 24: BU spectrometers spectral resolution over the 245 - $853 \mathrm{~nm}$ spectral range, and at different steps of the qualification tests. Credit: SuperCam. 
Transmission spectrometer gain and timing. The intensifier gain is a function of the high voltage power for the microchannel plate. The characterization was done, so that we can command the gain depending on the investigation (LIBS or TRR/L). Also, the intensifier gate timing shall be adjusted to optimize the signal on the detector. This is done by acquiring a series of spectra while increasing the delay of the shutter. These on-grounds characterizations are mandatory to be able to adjust parameters value in-flight.
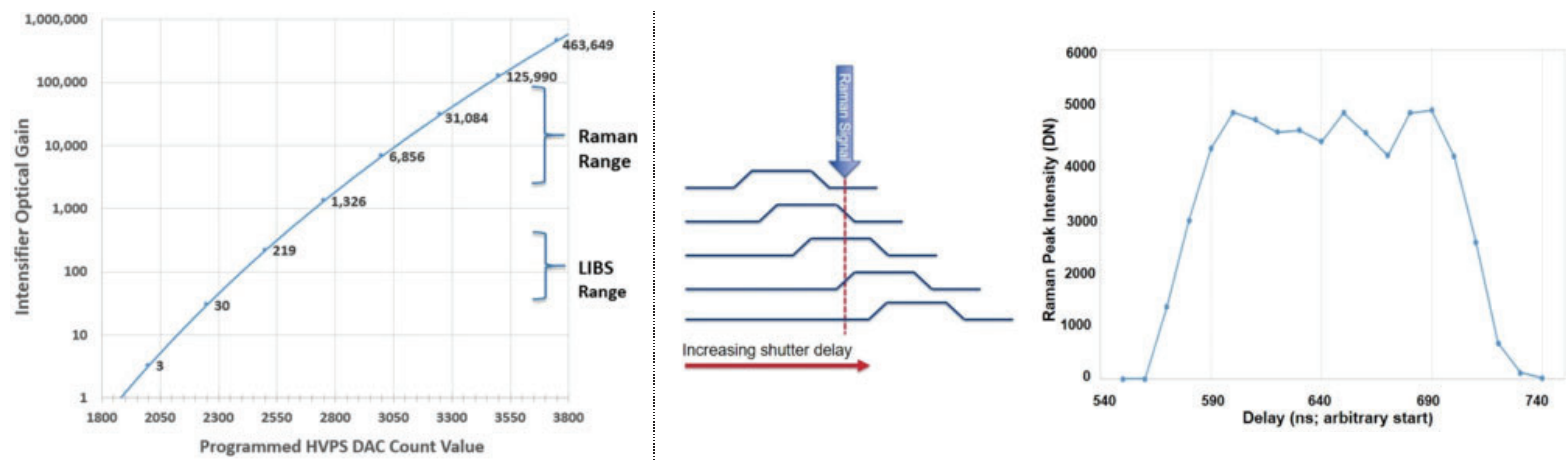

Figure 25: Left: characterization of the intensifier gain versus the high voltage command. Right: optimization of the intensifier gate timing to get the maximum of signal. The timing depends on the target distance. Credit: Wiens et al. (2020).

\subsection{End-to-end tests on Perseverance}

All SuperCam functions and techniques were exercised, and performance checked at instrument level. The data were retrieved from several test campaigns at JPL facilities, either at room temperature in ATLO (Assembly, Tests and Launch Operations), or in the huge thermal chamber.
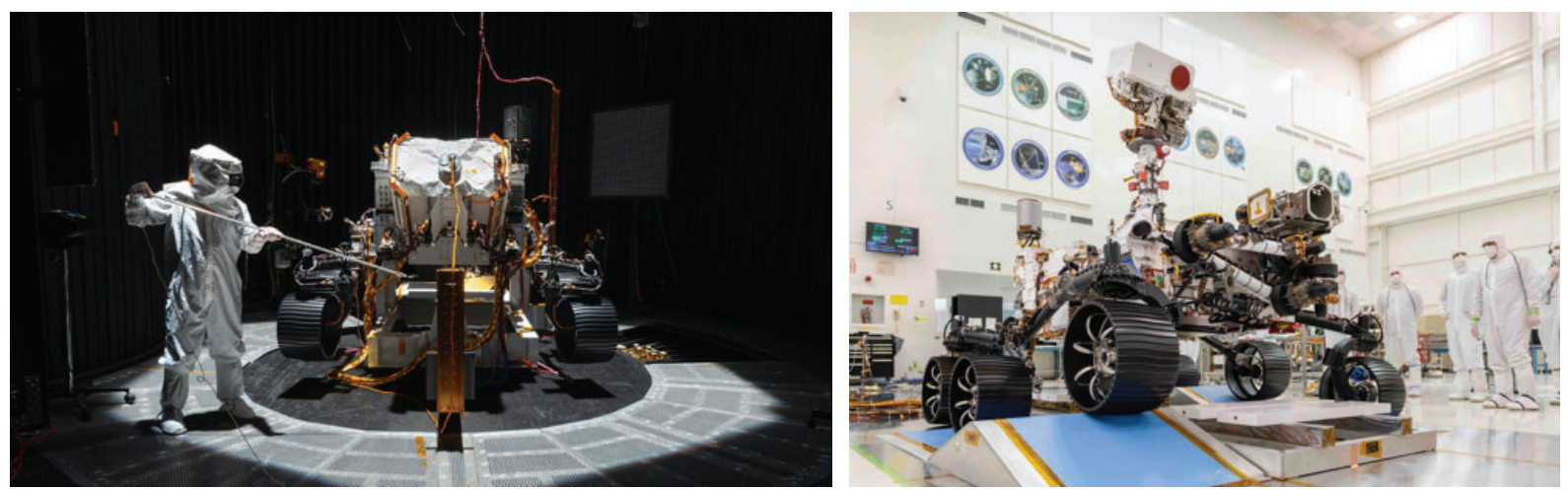

Figure 26: Perseverance rover installed in a thermal chamber at JPL (left). Perseverance tests in ATLO at JPL (right). Credit: NASA/JPL-Caltech.

Instrument Response Function. As for the infrared spectrometer, the instrument response function (IRF) is needed for the other spectroscopic techniques to invert the data and get calibrated spectra. ATLO tests were the first and only opportunity to measure the SuperCam IRF for the BU spectrometers, and thus for the LIBS and TRR/L. The team set up absolute calibrated sources on the ATLO floor, and recorded a series of spectra with Perseverance mast pointing at these sources. Preliminary IRF curves were obtained. 


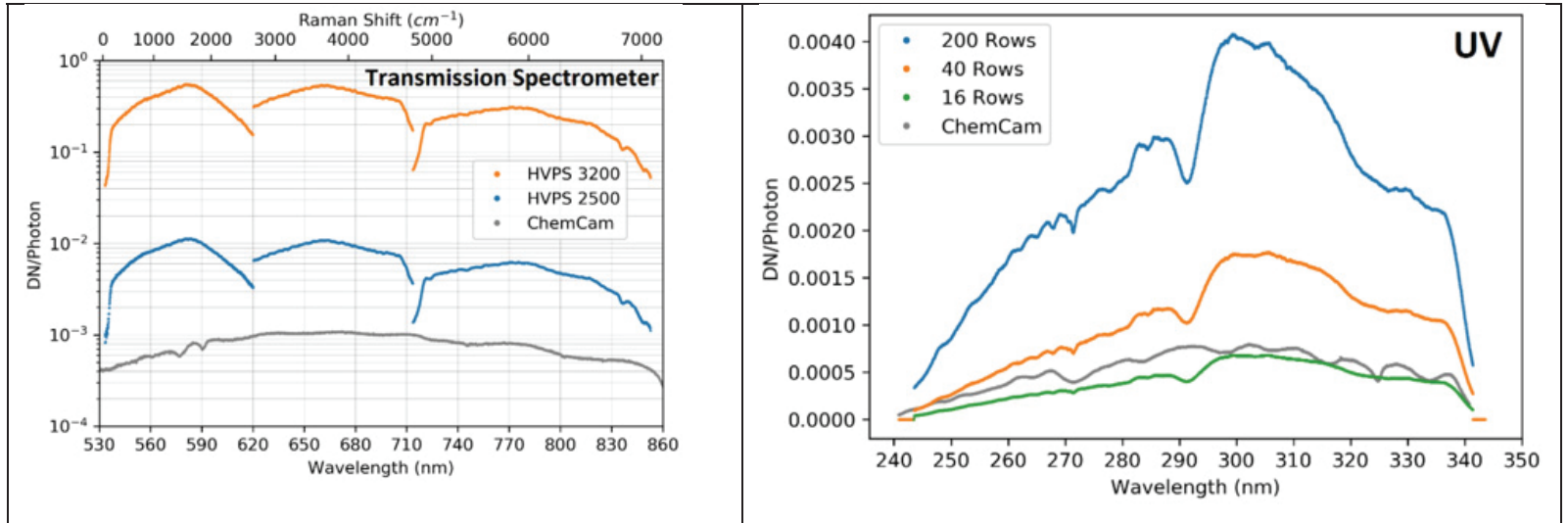

Figure 27: Preliminary instrument optical response functions for the transmission (left) and UV (right) spectrometers. The units are digital numbers (DN) per photon incident at the telescope aperture. Data were taken with the instrument mounted in the rover. For the reflection (UV, VIO) spectrometers, several read-out configurations (number of CCD rows) were tested to avoid saturation. For the transmission spectrometer (left), the intensifier gain depends on the techniques: 2500 for LIBS and 3200 for Raman spectroscopy. The response curves of the transmission spectrometer clearly show the three different (green, orange, red) diffraction orders. Credit: Wiens et al. (2020).

SuperCam techniques on Perseverance at cold. JPL proceeded to a two-week set of rover thermal tests under Martian pressure. The chamber was equipped with a solar simulator, and SuperCam team installed two sets of targets located at 2.6 $\mathrm{m}$ and $4.5 \mathrm{~m}$ from the rover mast. We were able to run the two autofocus modes, acquire RMI images, LIBS, TRR-L and VISIR spectra, and record sounds. The instrument behaved extremely well and end-to-end performance was demonstrated.
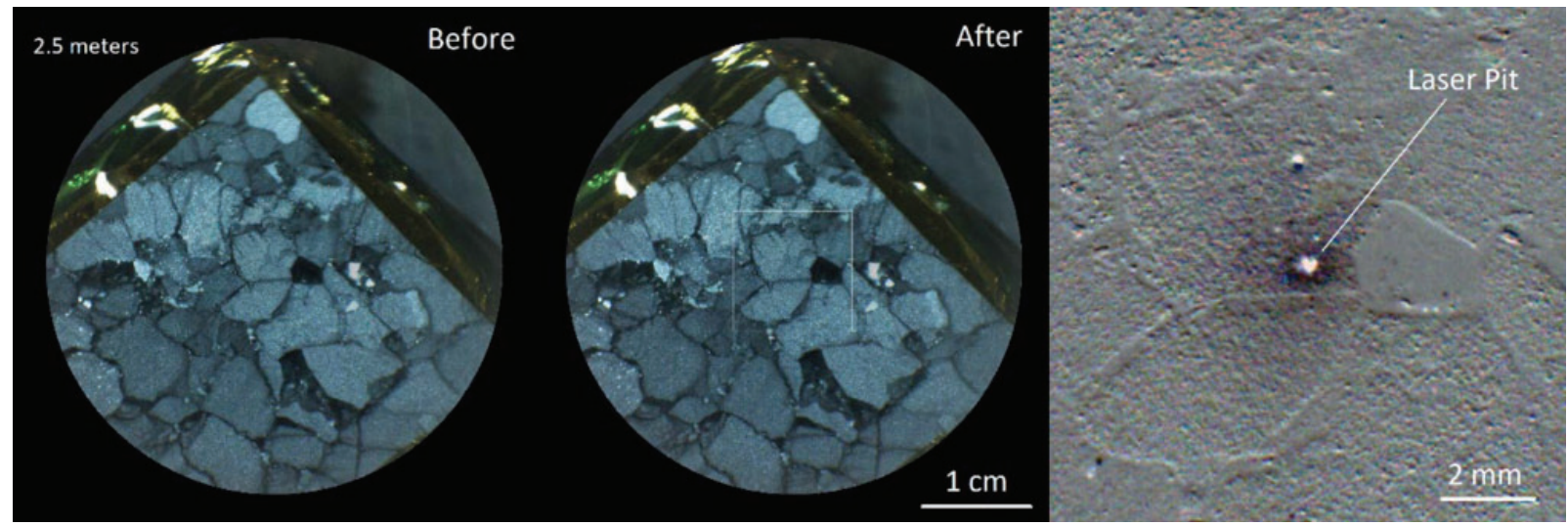

Figure 28: RMI images taken during STT of a composite target (62\% Ilmenite, 38\% Hematite) at $2.6 \mathrm{~m}$, using $19 \mathrm{~ms}$ integration time. (Left) Before LIBS, 30 shots. (Middle) After LIBS. (Right) Difference the two images to highlight the laser pit. This level of detail is expected of most RMI images taken in the rover's arm work zone and slightly beyond. Credit: Wiens et al. (2020).

LIBS, TRR/L and VISIR spectra are shown in Wiens et al. ${ }^{5}$

\section{CONCLUSION}

SuperCam was designed, manufactured, and tested in five years. The instrument performance assessed during the rover thermal test in October 2019 demonstrated that high-level science requirements are met.

Mars2020 mission was launched on July 30, 2020 from Cape Canaveral (Florida, US) for a seven months journey to Mars. During the cruise phase, SuperCam was powered ON two times to perform health checks: the different functions, including 
mechanisms actuation, thermal control, and detector dark measurements were tested, without firing the laser, which is forbidden when the rover mast is stowed.

Landing on Mars in scheduled on February 18, 2021. Surface transition operations will start just after landing, and SuperCam will acquire its first data on Sol 1. The first 15 Sols will be exclusively dedicated to technical activities to verify the instrument good health, followed by a phase of calibrations and first-time activities during another 75 Sols.

\section{TRIBUTE TO LAURENT PARES}

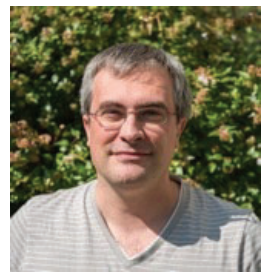

Laurent Parès was the optical designer for ChemCam on Curiosity, SuperCam on Perseverance, and many other space and on-ground instruments. He passed away on February 2020, leaving a great emptiness within the team. We lost a brilliant optical engineer and a friend.

\section{ACKNOWLEDGMENTS}

Funding in France was provided by the Centre National d'Etudes Spatiales (CNES). Human resources were provided in part by the Centre National de la Recherche Scientifique (CNRS) and universities. Funding was provided in the US by NASA's Mars Exploration Program. Funding in Spain was provided by the Spanish Science Ministry

Some funding of data analyses at Los Alamos National Laboratory (LANL) was provided by laboratory-directed research and development funds.

\section{REFERENCES}

[1] NASA Mars2020 mission and Perseverance rover, https://mars.nasa.gov/mars2020/

[2] Chide et al., "Recording laser-induced Sparks on Mars with the SuperCam microphone", Spectrochimica Acta Part B (2020), https://doi.org/10.1016/j.sab.2020.106000

[3] Chide et al., "Experimental Wind Characterization with the SuperCam Microphone under a Simulated martian Atmosphere", Icarus (2021), https://doi.org/10.1016/j.icarus.2020.114060

[4] Maurice, S. et al., "The SuperCam Instrument suite on the NASA Mars 2020 Rover: Science objectives and MastUnit Description”, Space Sci Rev (2021). SPAC-D-20-00069R1

[5] Wiens, R.C. et al., "The SuperCam Instrument Suite on the NASA Mars 2020 Rover: Body Unit and Combined System Tests", Space Sci Rev (2021) 217:4, https://doi.org/10.1007/s11214-020-00777-5

[6] Manrique, J.A. et al., "SuperCam Calibration Targets: Design and Development", Space Sci Rev (2021), https://doi.org/10.1007/s11214-020-00764-w

[7] Soujaeff, A. et al., New development for SuperCam Laser: UV conversion for spectroscopy and downsizing for compact LIBS instrument", this conference

[8] Gasnault, O. et al., "What SuperCam will see: The Remote Micro-Imager", Lunar and Planet. Sci. Conf. 52nd, abstract 2248, Houston, Texas, March 15-19, 2021.

[9] Reess, JM et al., "The SuperCam infrared instrument on the NASA Mars2020 mission: optical design and performance", Proceedings Volume 10562, International Conference on Space Optics — ICSO 2016; 105622J (2017), https://doi.org/10.1117/12.2296217

[10] Fouchet, T. et al., "The SuperCam Infrared Spectrometer for the Perseverance Rover of the Mars2020 mission", Icarus (2021), in preparation.

[11] Royer, C. et al., "Pre-launch radiometric calibration of the infrared spectrometer onboard SuperCam for the Mars2020 rover", Review of Scientific Instruments 91, 063105 (2020); https://doi.org/10.1063/1.5145390 